\title{
Indicações e Resultados das Intervenções Percutâneas em Artérias Carótidas
}

\author{
Rogério Tadeu Tumelero', Norberto Toazza Duda', Alexandre Pereira Tognon'
}

\section{RESUMO}

Neste artigo, os autores discutem a importância epidemiológica da doença oclusiva das artérias carótidas, destacando a apresentação clínica e os principais métodos para o seu diagnóstico, invasivos ou não. Além disso, discutem os principais estudos disponíveis na literatura comparando os resultados da endarterectomia carotídea aos das intervenções percutâneas.

DESCRITORES: Estenose das carótidas, terapia. Estenose das carótidas, cirurgia. Endarterectomia das carótidas. Doenças das artérias carótidas.

A s doenças cerebrovasculares são a terceira causa de morte nos EUA e o acidente vascular cerebral (AVC) é a causa mais comum de comprometimento neurológico na população idosa mundial ${ }^{1,2}$. Aproximadamente 750.000 pessoas têm AVC anualmente, com custo estimado de 45 bilhões de dólares em tratamento e redução na produtividade ${ }^{3,4}$. A doença oclusiva das artérias carótidas é responsável por 25\% desses $\mathrm{AVCs}^{5}$. Nos estudos de prevalência, a estenose nas artérias carótidas é de, aproximadamente, 0,5\% após os 60 anos de idade, com acréscimo de 10\% em pessoas com idade superior a 80 anos. A maioria dos casos é assintomática ${ }^{5,6}$. A cirurgia de endarterectomia é aceita como tratamento padrão para revascularização de lesões oclusivas extracranianas ${ }^{7}$, validado por meio de estudos randomizados e controlados, com demonstração da superioridade em relação ao tratamento medicamentoso. Entretanto, nos últimos anos, a utilização de stents para o tratamento das estenoses nas artérias carótidas tem surgido como terapêutica alternativa à endarterectomia. A terapia endovascular vem confirman-

${ }^{1}$ Cardiologia Intervencionista do Hospital São Vicente de Paulo, Passo Fundo, RS.

Correspondência: Rogério Tadeu Tumelero. Rua Teixeira Soares, 777/ 705 - Passo Fundo - RS. Fone: (54) 9973-3433 - Fax: (54) 3313-7203 E-mail: rttumelero@terra.com.br

Recebido em: 26/06/2006 - Aceito em: 21/12/2006

\section{SUMMARY}

Indication and Results of Percutaneous Interventions in Carotid Arteries

In this article, the authors discuss the epidemiological importance of carotid occlusive disease, highlighting the clinical presentation and the main methods for its diagnosis, both invasive and non-invasive. Additionally, the authors discuss the chief studies available in the literature, comparing the results of carotid endarterectomy to percutaneous interventions.

DESCRIPTORS: Carotid stenosis, terapy. Carotid stenosis, surgery. Endarterectomy, carotid. Carotid artery diseases.

do-se, por meio de estudos randomizados, como terapia igualmente eficaz à endarterectomia.

\section{Patologia}

No Framinghan Heart Study, o tipo mais comum de AVC foi infarto cerebral, responsável por $61 \%$ de todos os casos, excluindo-se os ataques isquêmicos transitórios (AITs). No National Heart Lung, and Blood Institute's Aterosclerosis Risk in Communities Study ${ }^{8}, 83 \%$ de todos os AVCs foram isquêmicos, 38\%, lacunar e 14\%, tromboembólico. Os locais mais comuns de obstrução são as bifurcações das artérias carótidas, a carótida interna e terço proximal das artérias vertebrais ${ }^{9,10}$.

\section{Apresentação clínica}

As manifestações da doença obstrutiva das artérias carótidas são o sopro carotídeo e os sintomas devido à isquemia cerebral. Entretanto, o paciente pode ter estenose grave sem sinais ou sintomas.

\section{a. Sopro carotídeo}

Quando presente, é um importante sinal de doença obstrutiva das artérias carótidas. Numa análise dos pacientes incluídos no estudo NASCET (North American Symptomatic Carotid Endarterectomy Trial) ${ }^{11}$, o sopro ipsilateral focal tem sensibilidade de $63 \%$ e especificidade de $61 \%$ para estenose grave, com maior resultado em pacientes sintomáticos, $75 \%$ dos quais possuíam estenose moderada a importante no teste diagnóstico. 


\section{b. Isquemia cerebral}

Pacientes com doença obstrutiva das artérias carótidas são diagnosticados clinicamente por meio de AITs $(<24 \mathrm{~h})$, déficits isquêmicos reversíveis (1 dia a 3 semanas), AVCs, amaurose fugaz ou afasia, nenhum específico para doença arterial carotídea. Após o diagnóstico de AIT, o risco do paciente apresentar AVC é $8 \%$, no primeiro mês, $12 \%$, primeiro ano, e maior que $30 \%$, nos 5 anos seguintes ${ }^{12}$.

\section{Diagnóstico}

O diagnóstico de obstrução das artérias carótidas, além da história e exame físico, necessita de um ou mais exames não-invasivo ou invasivo, como segue abaixo.

\section{A. Exames não-invasivos}

\section{- Ultra-sonografia}

É o exame não-invasivo mais usado para detectar as estenoses das artérias carótidas, incluindo a artéria carótida comum, a bifurcação e os ramos interno e externo. Utiliza os parâmetros de velocidade de fluxo, estimando, assim, o grau de estenose.

A ultra-sonografia com Doppler (US-Doppler), além de ser segura, por ser não-invasiva, é portátil e de baixo custo, podendo ser usada para detecção da composição da placa aterosclerótica.

Huston et al. ${ }^{13}$ compararam a US-Doppler com a angiografia convencional, em 1218 artérias carótidas. Para pacientes que tinham uma estenose de artéria carótida interna de $70 \%$ a $99 \%$, achados de um pico de velocidade sistólica de $>230 \mathrm{~cm} / \mathrm{s}$ tiveram uma sensibilidade de $86,4 \%$, uma especificidade de $90,1 \%$, um valor preditivo positivo de $82,7 \%$ e um valor preditivo negativo de 92,3\%. Uma velocidade diastólica final de $>70 \mathrm{~cm} / \mathrm{s}$ e um índice de artéria carótida de $>3,2$ tiveram valores semelhantes.

Entretanto, a US-Doppler tem limitações. Ela não é tão precisa em determinar estenoses de 50\% ou menos. Pode avaliar a porção extracranial das artérias carótidas, mas não a circulação cerebral intracraniana. Com a US-Doppler pode ser difícil distinguir entre uma artéria ocluída e uma estenose muito importante. De fato, o risco de classificar erroneamente uma estenose muito importante como ocluída tem sido relatado como sendo tão alto quanto $14 \%{ }^{14}$.

Finalmente, a acurácia da US-Doppler depende muito da experiência do ultra-sonografista, e pode ter consideráveis variações entre laboratórios.

\section{- Angiografia por ressonância magnética}

A angioressonância tem se tornado uma ferramenta valiosa na avaliação das estenoses das artérias carótidas. Uma vantagem importante é a possibilidade de visibilização de toda a extensão da circulação carotídea, desde sua origem até os vasos intracranianos. Uma imagem reproduzível de angioressonância de artérias carótidas tem uma sensibilidade de $75 \%$ a $90 \%$ em detectar estenoses de $70 \%$ a $99 \%{ }^{15-17}$.

Os resultados da angioressonância são menos confiáveis quando o fluxo sanguíneo é lento, turbulento ou ausente, e, como conseqüência, o grau da estenose é consistentemente superestimado, reduzindo a especificidade.

A angioressonância é menos operador-dependente que a US-Doppler, mas, consideravelmente, mais cara e não prática em pacientes criticamente doentes, em pacientes que têm implantes (como marca-passo) metálicos ou em pacientes claustrofóbicos.

\section{- Angiografia por tomografia computadorizada}

A angiotomografia pode obter imagens da vasculatura cervical e intracraniana; é capaz de distinguir oclusões de lesões de alto grau e é sensível e específica para diagnosticar estenoses carotídeas de alto grau quando comparada à US-Doppler ${ }^{18}$. Ao contrário da US-Doppler, a necessidade do uso de contraste pode ser uma contra-indicação ao seu uso, como no caso de pacientes com insuficiência renal.

\section{- Doppler transcraniano}

Pode ser usado em conjunto com a US-Doppler para identificar o fluxo de vasos colaterais intracranianos e avaliar o significado hemodinâmico das estenoses de carótidas extracraniais.

\section{- "Power" Doppler}

A imagem por "power" doppler é uma nova técnica sonográfica e tem aplicação vascular porque pode claramente definir superfícies intravasculares, e parece ser altamente sensível quando há baixo fluxo sanguíneo. Em um estudo feito por Steinke et al. ${ }^{19}$, imagens obtidas do lumen intravascular foram consideradas "boas" ou "excelentes" em comparação às visualizadas pelo Doppler com fluxo a cores (92\% vs 79\%).

\section{B. Exames invasivos}

\section{- Angiografia convencional com contraste}

Desde sua introdução, a angiografia com contraste tem sido o padrão-ouro para obter imagens de artérias carótidas. Recentes melhorias, como o uso de cateteres menores e macios, a técnica de Seldinger e a abordagem transfemoral, têm reduzido complicações e melhorado, significativamente, a resolução das imagens.

Todos os grandes trials de endarterectomias têm confiado na angiografia convencional para identificar candidatos a tal procedimento, pois este método visualiza tanto a vasculatura carotídea extracraniana como a intracraniana, incluindo os ramos do Polígono de Willis. Também visualiza a circulação vértebro-basilar e, ainda, 
Tumelero RT, et al. Indicações e Resultados das Intervenções Percutâneas em Artérias Carótidas. Rev Bras Cardiol Invas 2007; 15(2): 151-159.

fornece informações sobre a estrutura e possível ulceração ou dissecção da placa.

Sua desvantagem é a invasividade. O NASCET (North American Symptomatic Carotid Endarterectomy Trial) ${ }^{11}$ e outros estudos prospectivos relataram um risco de $4 \%$ para complicações neurológicas totais e de $1 \%$ para o risco de AVC ou morte ${ }^{20,21}$.

Embora invasiva e de risco, a angiografia convencional com contraste é o padrão-ouro para diagnosticar doenças das artérias carótidas. Entretanto, devido à sua associação com morbidade e mortalidade, ela pode ser precedida pelos testes não-invasivos.

Muitos estudos têm comparado a sensibilidade, especificidade e a acurácia da angiografia convencional com a US-Doppler, a angioressonância e com a angiotomografia. Em um estudo com 42 pacientes, Riles et al. ${ }^{22}$ relataram que a angioressonância se correlaciona exatamente com a angiografia convencional em 52\% dos pacientes e que a US-Doppler se correlaciona com a angiografia convencional em $65 \%$ dos pacientes. Ambos, US-Doppler e angiorressonância, mostraram superestimar o grau de estenose comparados à angiografia convencional (Tabela 1).

\section{Endarterectomia carotídea}

Em 1953, DeBakey ${ }^{23}$ realizou a primeira endarterectomia carotídea com sucesso para o tratamento de uma artéria carótida cervical ocluída. Em 1954, Eastcott et al. realizaram a primeira endarterectomia carotídea com sucesso, na qual a circulação cerebral foi intencionalmente interrompida para remover uma placa estenótica ${ }^{24,25}$. Apesar de dispor somente de evidências de eficácia provenientes de relatos de casos, aproximadamente 1 milhão de endarterectomias foram feitas no mundo inteiro entre 1974 e 1985 26,27. Houve um declínio temporário na metade dos anos 1980, quando vários relatos sugeriram inaceitáveis taxas de morte ou AVC perioperatórios $^{28-33}$, e uma alta taxa de endarterectomias realizadas sem indicações corretas ${ }^{34}$. Os estudos têm demonstrado que a endarterectomia carotídea cirúrgica confere benefícios significativos sobre o melhor tratamento clínico em pacientes com estenose carotídea sintomática $\geq 70 \%$, e com menores, embora significantes, graus de benefícios em lesões sintomáticas de 50\% a $69 \%$ e lesões assintomáticas $\geq 60 \%$. Entretanto, algumas observações se aplicam. Os critérios de seleção de pacientes devem ser estritamente seguidos ${ }^{35}$. Em um recente estudo prospectivo de 184 pacientes consecutivos submetidos a endarterectomia carotídea, relatou-se que somente $49 \%$ destes procedimentos foram adequadamente indicados ${ }^{36}$. O cirurgião deve ter uma baixa taxa de complicação perioperatória para assegurar o total (ou qualquer) benefício do procedimento. Alguns autores têm ainda sugerido que equipes cirúrgicas forneçam dados de suas taxas de AVC ou morte dentro de 30 dias da cirurgia ajustados para a gravidade da doença carotídea e condições coexistentes, referenciando médicos e pacientes ${ }^{37}$.

Finalmente, há em andamento debates sobre o custo-efetividade da cirurgia em pacientes com estenose carotídea assintomáticos, o que, de acordo com alguns autores, não está provado ${ }^{38}$.

\section{Pacientes de alto risco}

Os estudos NASCET, ACAS (Asymptomatic Carotid Atherosclerosis Study) e VA (Asymptomatic Carotid Stenosis Veterans Affairs) excluíram pacientes com comorbidades significativas, como insuficiência renal, hepática ou pulmonar, angina instável e infarto agudo do miocárdio (IAM) recente.

Em análise retrospectiva de 3111 endarterectomias consecutivas feita por Sundt et al. ${ }^{39,40}$, os pacientes foram estratificados em seis classes de risco, de acordo com o estado neurológico, comorbidades e variáveis angiográficas, revelando uma menor taxa de complicações (AVC incapacitante, IAM ou morte) para as classes I e II, enquanto a classe IV teve risco de complicações de $8,1 \%$ e de mortalidade de 2,9\% (Tabelas 2 e 3 ).

Fatores relacionados ao aumento da morbidade e mortalidade cirúrgica incluem estado neurológico instável; presença de comorbidades; idade acima de 70 anos; e oclusão de carótida interna contralateral.

Estes et al. ${ }^{41}$ acompanharam pacientes de uma amostra randomizada de 22.165 beneficiários de planos de saúde, que foram submetidos a endarterectomia carotídea entre 1988 e 1990, até 1992. Eles identifi-

TABELA 1

Comparação entre US Doppler (CDUS), Angioressonância (MRA), Angiografia (CCA) ${ }^{22}$

\begin{tabular}{lccc}
\hline & CDUS & MRA & CCA \\
\hline Sensibilidade & $86 \%$ & $94 \%$ & Padrão-Ouro \\
Especificidade & $90 \%$ & $86 \%$ & Padrão-Ouro \\
Custo & Baixo & Alto & Alto \\
Contra-indicações & Nenhuma & Implantes de Metal & Disfunção Renal; \\
absolutas e relativas & & Claustrofobia & acesso vascular difícil; ICC \\
Risco de AVC & $0 \%$ & $0 \%$ & $1 \%$ \\
\hline
\end{tabular}


TABELA 2

Classificação de Sundt et al. ${ }^{39}$, baseada na análise retrospectiva de 3111 pacientes submetidos a endarterectomia carotídea de maneira consecutiva

\begin{tabular}{|c|c|c|}
\hline $\begin{array}{c}\text { Classe } \\
\text { Sundt et al. }{ }^{39}\end{array}$ & Critérios para classificação & $\begin{array}{c}\text { Morbidade cirúrgica } \\
\text { combinada (Mortalidade)\% }\end{array}$ \\
\hline I & $\begin{array}{l}\text { Neurologicamente estável, ausência de risco maior clínica/ } \\
\text { angiograficamente definido, com doença estenótica-ulcerativa } \\
\text { unilateral/bilateral }\end{array}$ & 0,9 \\
\hline$\|$ & $\begin{array}{l}\text { Neurologicamente estável, ausência de risco clínico maior, } \\
\text { com ou sem risco angiográfico }\end{array}$ & 1,7 \\
\hline III & $\begin{array}{l}\text { Neurologicamente estável, presença de risco clínico maior, } \\
\text { com ou sem risco angiográfico }\end{array}$ & $3,7(1,3)$ \\
\hline IV & Neurologicamente instável, com ou sem risco clínico/angiográfico & $8,1(2,9)$ \\
\hline V & $\begin{array}{l}\text { Oclusão aguda da carótida interna, déficit neurológico progressivo } \\
\text { nas } 6 \text { h de avaliação }\end{array}$ & Não incluído no exame \\
\hline $\mathrm{VI}$ & Estenose carotídea sintomática recorrente & Não incluído no exame \\
\hline
\end{tabular}

TABELA 3

Definição de risco clínico, neurológico e angiográfico de Sundt et al ${ }^{39}$ para cirurgia da artéria carótida

\begin{tabular}{ll}
\hline Risco & Definição \\
\hline Clínico & $\begin{array}{l}\text { Doença arterial coronariana (angina, IAM }<6 \text { meses, ICC), hipertensão }(>180 / 110 \mathrm{mmHg}) \text {, doença vascular } \\
\text { periférica avançada, DPOC, idade }>70 \text { anos, obesidade acentuada }\end{array}$ \\
Neurológico & $\begin{array}{l}\text { Déficit neurológico em } 24 \mathrm{~h}, \text { isquemia cerebral geral, AVC }<7 \text { dias, AITs } \\
\text { Angiográfico }\end{array}$ \\
$\begin{array}{l}\text { Oclusão da carótida contralateral, estenose do sifão, placa }>3 \mathrm{~cm} \text { distalmente na carótida interna ou }>5 \mathrm{~cm} \\
\text { proximal na carótida comum, bifurcação na vértebra C2, pescoço curto e trombo mole estendendo-se de } \\
\text { uma lesão ulcerativa }\end{array}$ \\
\hline
\end{tabular}

IAM= infarto agudo do miocárdio; ICC= insuficiência cardíaca congestiva; DPOC= doença pulmonar obstrutiva crônica; AVC= acidente vascular cerebral; AIT= ataque isquêmico transitório.

caram pacientes com IAM, insuficiência cardíaca congestiva, diabete melito e idade acima dos 80 anos, como tendo taxas de sobrevida perioperatória e a longo prazo diminuídas após endarterectomia carotídea. Estenose carotídea recorrente também tem sido um indicador de alto risco de complicações de endarterectomias. Meyer et al. ${ }^{42}$ relataram uma taxa de morbidade e mortalidade perioperatórias de 10,8\%, em uma série de 82 pacientes submetidos a endarterectomia carotídea para estenose recorrente; isto foi cinco vezes o risco de endarterectomia de rotina na instituição dos autores. Lesões nos nervos cranianos e cervicais podem ocorrer em $21 \%$ dos pacientes, embora ocorram poucas lesões permanentes ${ }^{43}$.

Gasparis et al. ${ }^{44}$ estratificaram pacientes submetidos a endarterectomia em baixo e alto risco, baseados na idade acima dos 80 anos, angina pela NYHA (New York Heart Association) classes III/IV, insuficiência cardíaca classes III/IV pela Canadian, IAM nos últimos 6 meses, doença pulmonar oxigênio ou corticóide dependente, creatinina $\geq 3$, oclusão de carótida contralateral, bifurcação carotídea alta, reoperação ou irradiação cervical. O risco de AVC ou morte perioperatório foi $1,3 \%$, no grupo de alto risco, e $1,1 \%$, no grupo de baixo risco (não significativo).

\section{Tratamento endovascular de doença carotídea oclusiva}

O tratamento endovascular (angioplastia e stent) de doença carotídea oclusiva oferece vantagens sobre a endarterectomia: anestesia geral é geralmente não necessária, permitindo que o estado clínico neurológico do paciente seja monitorado durante o procedimento; aumento do conforto do paciente e significativa economia devido ao curto período de recuperação; nenhuma incisão cervical é feita, portanto, eliminando o risco de paralisias de nervos cranianos, infecções de ferida operatória ou hematoma cervical; os procedimentos podem ser feitos simultaneamente na carótida, vertebral e artérias coronárias; morbidade e mortalidade podem ser reduzidas em pacientes considerados de alto risco para cirurgia (que têm significantes comorbidades, oclusão de carótida contralateral, reestenose pós-endarterectomia, estenose induzida por radiação, dissecção 
Tumelero RT, et al. Indicações e Resultados das Intervenções Percutâneas em Artérias Carótidas. Rev Bras Cardiol Invas 2007; 15(2): 151-159.

cervical prévia); e ótima opção para pacientes com lesões inacessíveis cirurgicamente.

\section{- Angioplastia carotídea por balão}

Angioplastia transluminal percutânea por balão para estenose de carótida foi primeiro relatada por Kerber et al. ${ }^{45}$, em 1980. Em 1987, Theron et al. ${ }^{46}$ publicaram a primeira grande série de angioplastia de carótida interna, em 48 pacientes com aterosclerose de novo ou reestenose pós-cirurgia. Sucesso técnico foi atingido em $94 \%$ dos casos, com uma taxa de $4,1 \%$ de morbidades sérias. Gil-Peralta et al. ${ }^{47}$ realizaram 85 angioplastias por balão, em 82 pacientes com estenose carotídea $>70 \%$ sintomáticos, num período de quatro anos. Eles obtiveram sucesso técnico em 92\%, com mortalidade em 30 dias de $0 \%$, e taxa de morbidades maiores de $4,9 \%$, as quais se comparam favoravelmente com os resultados do ECST (European Carotid Surgery Trial) e NASCET.

Apesar desses resultados favoráveis, a angioplastia por balão tem um número potencial de limitações, incluindo retração elástica da parede do vaso, dissecção intimal evidente angiograficamente e descolamento de placas e partículas de embolização. Angioplastia de lesões ateroscleróticas tem sido relatada como possível geradora de êmbolos, compostos de ateroma, cristais de colesterol, trombo e agregados plaquetários. O risco de dano cerebral pensa-se ser dependente do tamanho e da composição do material embólico, bem como da extensão e do local do cérebro envolvidos. Devido à dificuldade em distinguir-se precisamente entre ar e partículas embólicas usando doppler transcraniano, a falta de correlação entre êmbolo e seqüela clínica levou a sugerir que a maioria dos êmbolos detectados durante angioplastia por balão é gasosa ou constituída por pequenos agregados plaquetários de $<200 \mu \mathrm{m}$ de diâmetro, e ambos se correlacionam com desfechos mais benignos ${ }^{48-51}$.

\section{- Stent carotídeo}

O ímpeto para o uso de stent carotídeo tem crescido principalmente pelos estudos de stent versus simples angioplastia por balão em artérias coronárias, que têm consistentemente demonstrado um persistente benefício em sobrevida livre de eventos em um ano e uma menor taxa de angioplastias repetidas ${ }^{52-54}$. As vantagens do stent sobre a angioplastia simples incluem evitar o deslocamento da placa, a dissecção da íntima e a retração elástica arterial, além de reduzir reestenose tardia.

\section{- Séries de Casos e Estudos Randomizados}

Desde 1996, houve 11 grandes séries publicadas de stents carotídeos, com um número total de 1311 pacientes ${ }^{55-66}$. A taxa de sucesso técnico relatada foi $>95 \%$; taxas de mortalidade relacionadas ao procedimento (incluindo mortes cardíacas) são de 0,6\% a 4,5\%; taxas de AVC maior de $0 \%$ a $4,5 \%$; taxas de
AVC menor de $0 \%$ a $6,5 \%$; e taxa de reestenose em 6 meses $<5 \%$. Estes estudos excluem os outros que representam coortes de muito alto risco ${ }^{63,64,67-70}$.

Wholey e Eles $^{71}$ publicaram os resultados de um estudo mundial utilizando stent carotídeo, no qual 3047 procedimentos endovasculares foram realizados em 24 centros na América do Norte, Europa, Ásia e América do Sul. Eles relataram uma taxa de mortalidade em 30 dias relacionada ao procedimento de 0,98\%, taxas de AVC maior de 1,35\%, taxas de AVC menor de $2,53 \%$, e uma taxa de reestenose em 6 e 12 meses de $2,23 \%$ e $2,48 \%$, respectivamente. Centros que trabaIharam com menos de 50 casos tiveram 6,4\% de AVC maior e morte comparados com 2,3\% para centros que trabalharam com 50 e 100 casos. Os autores então sugeriram uma curva de aprendizado de 50 casos para stent carotídeo.

Esta série de casos também analisou desfechos para pacientes considerados de alto risco para cirurgia. No estudo NASCET ${ }^{72}$, a taxa de morte e AVC perioperatório foi de $5,8 \%$, com $0,6 \%$ da taxa de mortalidade causada por IAM. Os critérios do NASCET foram aplicados para pacientes em cinco coortes desta série de stent em carótidas, demonstrando que 79\% destes 574 pacientes teriam falência de elegibilidade devido as comorbidades $56,60,61,64$. Apesar disso, taxas de morbidade de $2 \%$ a $7,9 \%$ e taxas de mortalidade de $0,6 \%$ a $2 \%$ para estes precoces relatos de stents carotídeos, se comparam favoravelmente aqueles no NASCET e no ECST. Entretanto, 41\% $(n=233)$ destes pacientes tinham estenose de carótida e eram assintomáticos, para os quais menores taxas de mortalidade e morbidade para a endarterectomia seriam esperados.

Há poucos estudos publicados comparando diretamente endarterectomia carotídea com stent carotídeo. Jordan et al. ${ }^{73}$ compararam, retrospectivamente, 107 pacientes endarterectomizados com 166 pacientes seguidos prospectivamente submetidos a stent carotídeo e relataram uma maior taxa de AVC menor precoce no grupo stent $(6,6 \%$ vs $0,6 \%)$, mas uma maior taxa de AVC maior e morte nos pacientes cirúrgicos $(4,2 \%$ vs $2,8 \%)$.

Revendo a literatura de stent carotídeo, a taxa de sucesso técnico, taxa de morbidade e mortalidade relacionadas ao procedimento, e taxas de reestenose, mostramse comparáveis à da endarterectomia carotídea ${ }^{74-76}$. Entretanto, inconsistências em desenhos dos estudos, critérios relatados e seguimentos, fazem a comparação da endarterectomia com revascularização endovascular muito contestada. A validade das comparações entre tais dados clínicos desiguais é não confiável.

O estudo Carotid and Vertebral Transluminal Angioplasty Study (CAVATAS) ${ }^{77}$, multicêntrico, prospectivo e randomizado, comparou endarterectomia carotídea com angioplastia carotídea. Foram randomizados 504 pacientes para cirurgia e angioplastia, num período de cinco anos. Não houve diferença significativa no risco de AVC ou 
morte relacionados ao procedimento entre endarterectomia carotídea e angioplastia. Análises preliminares de sobrevida a longo prazo não mostraram diferenças na taxa de AVC ipsilateral ou qualquer AVC incapacitante, seguidos em três anos após a randomização.

O Wallstent Trial ${ }^{8}$ foi um estudo financiado pela indústria, prospectivo, randomizado, comparando endarterectomia e stent carotídeo para estenoses $\geq 60 \%$, sintomáticos. Neste estudo, 219 pacientes foram randomizados para cirurgia ou stent. O risco de qualquer AVC perioperatório ou morte foi de 4,5\% para cirurgia e $12,1 \%$ para o stent. Em um ano, o risco de AVC maior foi $0,9 \%$ para cirurgia e $3,7 \%$ para o stent. Este estudo foi interrompido precocemente devido aos resultados do stent.

O estudo Carotid Revascularization Endarterectomy versus Stent Trial (CREST) ${ }^{79,80}$, um ensaio clínico norteamericano, multicêntrico, randomizado e controlado, comparou a eficácia da endarterectomia cirúrgica com o stent carotídeo, e ainda está em andamento. Para demonstrar diferença clínica significativa entre os dois tratamentos, o estudo precisará de 2500 pacientes, excluindo-se comparações entre vários subgrupos clínicos.

O problema da embolização distal durante angioplastia com balão e stent carotídeo tem aumentado o interesse em fornecer métodos para proteção cerebral. Theron et al. ${ }^{55,81}$ relataram complicações embólicas distais em 3 de 38 pacientes ( $8 \%$ ) submetidos a angioplastia por balão de carótida interna sem protetor distal versus 0 de 43 pacientes $(0 \%)$ submetidos ao mesmo procedimento usando protetor distal. Henry et al. ${ }^{82}$ usaram a técnica de oclusão distal do balão de Theron em 32 de 163 casos de stent carotídeo. Entretanto, 2 de 3 AVC maiores que ocorreram nesta série foram em conjunto com este dispositivo. Os autores citaram tempo de procedimento prolongado e aumento do risco de embolismo quando cruzam lesões ulceradas, como potenciais problemas associados ao seu uso.

O estudo Stenting and Angioplasty with Protection in Patients at High Risk for Endarterectomy (SAPPHIRE) ${ }^{83}$ é considerado um dos estudos randomizados, multicêntrico de maior significância comparando endarterectomia versus implante de stent com protetor de embolização distal. Entre agosto de 2000 e julho de 2002, 747 pacientes foram selecionados para o estudo, e 334 foram randomizados. Dos 413 pacientes que não foram randomizados porque tinham uma contraindicação para um dos tratamentos, 406 formaram um registro de stent e 7 um registro cirúrgico. Dos 167 pacientes randomizados para o grupo stent, 159 receberam o tratamento designado.

Foram selecionados pacientes sintomáticos com estenose carotídea de pelo menos 50\% e também pacientes assintomáticos com estenose de pelo menos $80 \%$ do diâmetro luminal.
O protetor de embolização distal foi usado com sucesso em 95,6\% dos pacientes randomizados para o stent.

O desfecho primário (incidência cumulativa de morte, AVC, ou IAM dentro de 30 dias após o procedimento ou morte ou AVC ipsilateral entre os dias 31 e 1 ano após) ocorreu em 20 dos 167 pacientes randomizados para receber o stent (incidência cumulativa de 12,2\%) e em 32 dos 167 pacientes randomizados para a cirurgia (incidência cumulativa de 20,1\%), com uma diferença absoluta de $-7,9$ pontos percentuais (intervalo de confiança de $95 \%,-16,4$ para 0,7 pontos percentuais; $\mathrm{p}=0,004$ para não-inferioridade).

A taxa estimada de paralisia de nervos cranianos em um ano foi maior entre aqueles submetidos a cirurgia do que aqueles que receberam stent $(4,9 \%$ vs $0 \%$, $p=0,004)$, e a taxa estimada de revascularização do vaso-alvo de $4,3 \%$ para endarterectomia vs $0,6 \%$ para o stent $(p=0,004)$.

No período periprocedimento (até 30 dias), a incidência cumulativa de AVC, IAM ou morte foi de 4,8\% entre os pacientes do grupo stent e de 9,8\% entre os pacientes do grupo submetido a endarterectomia, na análise intenção-de-tratar $(p=0,09)$, e de 4,4\% entre os pacientes que realmente receberam o stent e de 9,9\% entre aqueles que foram submetidos a endarterectomia $(p=0,06)$. A média de permanência no hospital foi de $1,84 \pm 1,75$ dias, entre os pacientes que receberam stent,

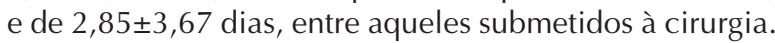

Para pacientes com estenose de carótida assintomáticos, a incidência acumulada do desfecho primário em um ano foi menor entre aqueles que receberam stent $(9,9 \%)$ do que entre aqueles submetidos a endarterectomia $(21,5 \% ; p=0,02)$. No período periprocedimento, a incidência cumulativa de morte, IAM, ou AVC entre pacientes com estenose assintomática, foi de $5,4 \%$, no grupo stent, e de $10,2 \%$, no grupo da endarterectomia $(p=0,2)$.

Os resultados deste estudo mostraram que o stent não foi inferior à cirurgia, e a taxa de desfecho primário (morte, AVC ou IAM, em 30 dias) foi 39\% menor entre os pacientes randomizados para receber o stent com protetor distal do que aqueles submetidos à endarterectomia.

O estudo Endarterectomy versus Stenting in Patients with Symptomatic Severe Carotid Stenosis (EVA-3S) ${ }^{84}$ arrolou 527 pacientes, evidenciando taxa de AVC ou morte em 30 dias de 3,9\%, no grupo tratado com endarterectomia, e de 9,6\%, naquele tratado com stent. Em seis meses, a taxa de AVC ou morte foi de 6,1\%, nos pacientes tratados com endarterectomia, e de 11,7\%, nos tratados com stent $(p=0,02)$.

Os resultados de 30 dias do estudo Stent-Protected Angioplasty versus Carotid Endarterectomy in Symptomatic Patients (SPACE) ${ }^{85}$, que arrolou 1200 pacientes, 
demonstraram taxa de óbito ou AVC isquêmico ipsilateral de $6,84 \%$, nos pacientes tratados com stent, e de $6,34 \%$, naqueles tratados com endarterectomia $(p=0,09)$, não permitindo confirmar a hipótese de não-inferioridade do implante de stent em relação à endarterectomia. Resultados tardios devem ser considerados para uma avaliação mais consistente.

\section{Seleção de pacientes para tratamento percutâneo}

Os pacientes podem ser selecionados de acordo com múltiplos fatores, como o grau de estenose, a presença de sintomas e a de fatores que colocam o paciente como de alto risco para endarterectomia. $\mathrm{O}$ grau de estenose é inicialmente medido com métodos não-invasivos, como doppler carotídeo, angioressonância ou angiotomografia.

Recomenda-se definir pacientes sintomáticos aqueles com sintomas neurológicos ou mudanças neurológicas dentro dos últimos seis meses (180 dias) do tratamento. Sintomas podem ser AVC prévio ou evento neurológico transitório. Eventos transitórios podem ser hemisféricos ou retinianos (amaurose). A população de pacientes pode ser classificada ainda em baixorisco ou alto-risco em relação à endarterectomia. É desconhecido se tais pacientes são também de altorisco se tratados com stent carotídeo.

Pacientes de baixo-risco são aqueles com fatores de risco similares, aos que foram inscritos nos estudos NASCET e ACAS. A definição de pacientes de alto-risco é mais controversa. Sugestões para categorizar pacientes de alto-risco que estão listadas na Tabela 2, baseados em critérios usados em estudos de stent prévios e também em critério de Sundt. Os pacientes de alto risco podem ser definidos como tendo $\geq 1$ fator de risco anatômico ou $\geq 2$ comorbidades do grupo $\mathrm{A}$ ou $\geq 1$ comorbidade do grupo B, como definidos na Tabela 3 .

\section{CONCLUSÃO}

Os resultados de angioplastia e stent carotídeos variam. Séries de casos mostram resultados comparáveis aos da endarterectomia, particularmente nos pacientes inelegíveis do estudo NASCET. Entretanto, dois grandes estudos prospectivos e randomizados têm resultados discordantes com o CAVATAS, mostrando a angioplastia e/ou stent com desfechos comparáveis à cirurgia e o WALLSTENT mostrando que o stent carotídeo tem desfechos inferiores aos da cirurgia, assim como o estudo EVA-3S. Já os resultados do estudo SAPPHIRE mostram que a angioplastia-stent com protetor distal é comparável à endarterectomia, nos pacientes de alto risco cirúrgico. O estudo SPACE, por sua vez, não confirmou a nãoinferioridade do implante de stent em relação à endarterectomia em 30 dias. Faz-se necessário, no entanto, aguardar os resultados tardios.

Diante destes resultados discordantes e das inconsistências na seleção dos pacientes e nas definições usadas para relatar os desfechos, relatos padrões são necessários, com modelos para o desenho do estudo. Isto permitirá a criação de estudos confiáveis de segurança e efetividade para a revascularização endovascular carotídea usando tecnologias atuais.

Em pacientes de alto risco cirúrgico com doença carotídea, a angioplastia com implante de stent com dispositivos de proteção distal é a terapia de escolha. Para pacientes de baixo risco cirúrgico, a endarterectomia cirúrgica continua sendo o tratamento de escolha.

A classificação dos pacientes com estenoses de artérias carótidas no grupo de alto risco cirúrgico, utilizando os critérios de Sundt, os referencia para tratamento percutâneo com implante de stent associado ao uso de protetor de embolização distal cerebral, proporcionando taxas de complicações iguais ou inferiores às da endarterectomia cirúrgica.

\section{REFERÊNCIAS BIBLIOGRÁFICAS}

1. The National Advisory Neurological Disorders and Stroke Council. Stroke and cerebrovascular disease. Bethesda:National Institutes of Health Report; 1992;26-7.

2. Bonita R, Beaglehole R. Stroke: populations, cohorts, and clinical trials. In: Whisnant JP, ed. Stroke mortality. Oxford: Butterworth-Heinemann;1993. p.59-79.

3. 1999 Heart and Stroke Statistical Update. Dallas:American Heart Association;1998. p.13-5.

4. Matchar D, Duncan P. Cost of stroke. Stroke Clinical Updates. 1994:9-12.

5. Ricci S, Flammi FO, Marini M, Antonini D, Bartolini S, Celani MG, et al. The prevalence of stenosis of the internal carotid in subjects over 49: a population study. Epidemiol Prev. 1991;13(48-49):173-6.

6. O'Leary DH, Polak JF, Kronmal RA, Kittner SJ, Bond $\mathrm{MH}$ Wolfsoc SK Jr., et al. Distribution and correlates of sonographically detected carotid artery disease in the Cardiovascular Health Study. The CHS Collaborative Research Group. Stroke. 1992;23(12):1752-60.

7. Zarins CK. Carotid endarterectomy: the gold standard. J Endovasc Surg. 1996;3(1):10-5.

8. Rosamond WD, Folsom AR, Chambless LE, Wang $\mathrm{CH}$, McGouern PG, Howard G, et al. Stroke incidence and survival among middle-aged adults: 9-year follow-up of the Atherosclerotic Risk in Communities (ARIC) cohort. Stroke. 1999;30(4):736-43.

9. Fischer CM, Gore I, Okabe N. Atherosclerosis of the carotid and vertebral arteries - extracranial and intracranial. J Neuropathol Exp Neurol. 1965;24(3):455-76.

10. Davies KN, Humphrey PR. Do carotid bruits predict disease of the internal carotid arteries? Posgrad Med J. 1994;70 (824):433-5.

11. Sauvé JS, Thorpe KE, Sackett DL, Taylor W, Barnett HJ, Haynes RB, et al. Can bruits distinguish high-grade from moderate symptomatic carotid stenosis? The North American Symptomatic Carotid Endarterectomy Trial. Ann Intern Med. 1994;120(8):633-7.

12. Sacco RL. Identifying patient populations at high risk for stroke. Neurology. 1998;51(3 suppl 3):S27-30.

13. Huston J $3^{\text {rd }}$, James EM, Brown RD Jr, Lefsrud RD, Ilstrup DM, Robertson EF, et al. Redefined duplex ultrasonographic criteria for diagnosis of carotid artery stenosis. Mayo Clin Proc. 2000;75(11):1133-40. 
14. Bridgers SL. Clinical correlates of Doppler/ultrasound errors in the detection of internal carotid artery occlusion. Stroke. $1989 ; 20(5): 612-5$.

15. Anderson CM, Saloner D, Lee RE, Gniswold VJ, Shapeer LG, Rapp JH, et al. Assessment of carotid artery stenosis by MR angiography: comparison with X-ray angiography and color-coded Doppler ultrasound. AJNR Am J Neuroradiol. 1992;13(3):989-1003.

16. Mattle HP, Kent KC, Edelman RR, Atkinson DJ, Skillman JJ. Evaluation of the extracranial carotid arteries: correlation of magnetic resonance angiography, duplex ultrasonography and conventional angiography. J Vasc Surg. 1991;13(6):838-45.

17. Polak JF, Bajakian RL, O'Leary DH, Anderson MR, Donaldson MC, Jolesz FA. Detection of internal carotid artery stenosis: comparison of MR angiography, color Doppler sonography and arteriography. Radiology. 1992;182(1):35-40.

18. Dillon EH, van Leeuwen MS, Fernandez MA, Eikelboom BC, Mali WP. CT angiography: application to the evaluation of carotid artery stenosis. Radiology. 1993;189(1):211-9.

19. Steinke W, Ries S, Artemis N, Schwartz A, Hennerici M. Power Doppler imaging of carotid artery stenosis. Comparison with color Doppler flow imaging and angiography. Stroke. 1997;28(10):1981-7.

20. Davies KN, Humphrey PR. Complications of cerebral angiography in patients with symptomatic carotid territory ischaemia screened by carotid ultrasound. J Neurol Neurosurg Psychiatry. 1993;56(9):967-72.

21. Eliasziw M, Rankin RN, Fox AJ, Haynes RB, Barnett HJ. Accuracy and prognostic consequences of ultrasonography in identifying severe carotid artery stenosis. North American Symptomatic Carotid Endarterectomy Trial (NASCET) Group. Stroke. 1995;26(10):1747-52.

22. Riles TS, Eidelman EM, Litt AW, Pinto RS, Oldford F, Schwartzenberg GW. Comparison of magnetic resonance angiography, conventional angiography, and duplex scanning. Stroke. 1992;23(3):341-6.

23. DeBakey ME. Carotid endarterectomy revisited. J Endovasc Surg. $1996 ; 3(1): 4$.

24. Eastcott $\mathrm{HH}$, Pickering GW, Rob CG. Reconstruction of internal carotid artery in a patient with intermittent attacks of hemiplegia. Lancet. 1954;267(6846):994-6.

25. Eastcott $\mathrm{HH}$. Late thoughts and reflections on carotid reconstruction for the prevention of ischemic stroke. J Endovasc Surg. 1996;3(1):5-6.

26. Barnett H. Symptomatic carotid endarterectomy trials. Stroke. 1991;21(11 suppl):III2-5.

27. Barnett $H$. Evaluating methods for prevention in stroke. Ann R Coll Physicians Surg Can. 1991;24(1):33-42.

28. Shaw DA, Venables GS, Cartlidge NE, Bates D, Dickinson $\mathrm{PH}$. Carotid endarterectomy in patients with transient cerebral ischaemia. J Neurol Sci. 1984;64(1):45-53.

29. Muuronen A. Outcome of surgical treatment of 110 patients with transient ischemic attack. Stroke. 1984;15(6):959-64.

30. Slavish LG, Nicholas GG, Gee W. Review of a community hospital experience with carotid endarterectomy. Stroke. 1984;15(6):956-9.

31. Brott T, Thalinger $\mathrm{K}$. The practice of carotid endarterectomy in a large metropolitan area. Stroke. 1984;15(6):950-5.

32. Warlow C. Carotid endarterectomy: does it work? Stroke. 1984;15(6):1068-76.

33. Barnett HJ, Plum F, Walton JN. Carotid endarterectomy: an expression of concern. Stroke. 1984;15(6):941-3.

34. Winslow CM, Solomon DH, Chassin MR, Kosecoff J, Merrick NJ, Brook RH. The appropriateness of carotid endarterectomy. N Engl J Med. 1988;318(12):721-7.
35. Marcinczyk MJ, Nicholas GG, Reed JF $3^{\text {rd }}$, Nastasee SA. Asymptomatic carotid endarterectomy. Patient and surgeon selection. Stroke. 1997;28(2):291-6.

36. Wong JH, Lubkey TB, Suarez-Almazor ME, Findlay JM. Improving the appropriateness of carotid endarterectomy: results of a prospective city-wide study. Stroke. 1999;30(1):12-5.

37. Chassin MR. Appropriate use of carotid endarterectomy. $N$ Engl J Med. 1998;339(20):1468-71.

38. Warlow C. Endarterectomy for asymptomatic carotid stenosis? Lancet. 1995;345(8960):1254-5.

39. Sundt TJ, Meyer F, Piepgras DG, Fode N, Ebesold M, Marsh W. Risk factors and operative results. Sundt's occlusive cerebrovascular disease. Philadelphia:WB Saunders;1994. p.241-7.

40. Sundt T, Sandok B, Whisnant JP. Carotid endarterectomy. Complications and preoperative assessment of risk. Mayo Clin Proc. 1975;50(6):301-6.

41. Estes JM, Guadagnoli E, Wolf R, LoGerfo FW, Whittemore AD. The impact of cardiac comorbidity after carotid endarterectomy. J Vasc Surg. 1998;28(4):577-84.

42. Meyer FB, Piepgras DG, Fode NC. Surgical treatment of recurrent carotid artery stenosis. J Neurosurg. 1994;80(5):781-7.

43. AbuRahma AF, Choueiri MA. Cranial and cervical nerve injuries after repeat carotid endarterectomy. J Vasc Surg. 2000;32(4):649-54

44. Gasparis AP, Ricotta L, Cuadra SA, Char DJ, Purtill WA, Van Bemmelen PS, et al. High-risk carotid endarterectomy: fact or fiction. J Vasc Surg. 2003;37(1):40-6.

45. Kerber CW, Cromwell LD, Loehden OL. Catheter dilatation of proximal carotid stenosis during distal bifurcation endarterectomy. AJNR Am Neuroradiol. 1980;1(4):348-9.

46. Theron J, Raymond J, Casasco A, Courtheoux F. Percutaneous angioplasty of atherosclerotic and postsurgical stenosis of carotid arteries. AJNR Am J Neuroradiol. 1987;8(3):495-500.

47. Gil-Peralta A, Mayol A, Marcos JR, Gonzales A, Ruano J Boza F, et al. Percutaneous transluminal angioplasty of the symptomatic atherosclerotic carotid arteries. Results, complications, and follow-up. Stroke. 1996;27(12):2271-3.

48. Bladin CF, Bingham L, Grigg L, Yapanis AG, Gerraty R, Davis SM. Transcranial Doppler detection of microemboli during percutaneous transluminal coronary angioplasty. Stroke. 1998;29(11):2367-70.

49. Markus HS, Clifon A, Buckenham T, Brown MM. Carotid angioplasty. Detection of embolic signals during and after the procedure. Stroke. 1994;25(12):2403-6.

50. Crawley F, Clifton A, Buckenham T, Loosemore T, Taylor RS, Brown MM. Comparison of hemodynamic cerebral ischemia and microembolic signals detected during carotid endarterectomy and carotid angioplasty. Stroke. 1997;28(12):2460-4.

51. McCleary AJ, Nelson M, Dearden NM, Calvey TA, Gough MJ. Cerebral haemodynamics and embolization during carotid angioplasty in high-risk patients. Br J Surg. 1998;85(6):771-4.

52. Serruys PW, de Jaegere $P$, Kiemeneij F, Macaya C, Rutsch W, Heyndrickx G, et al. A comparison of balloon- expandablestent implantation with ballon angioplasty in patients with coronary artery disease. Benestent Study Group. N Engl J Med. 1994;331(8):489-95

53. Macaya C, Serruys PW, Ruygrok P, Suryapranata H, Mast G, Klugmannn $\mathrm{S}$, et al. Continued benefit of coronary stenting versus balloon angioplasty: one-year clinical follow-up of Benestent trial. Benestent Study Group. J Am Coll Cardiol. 1996;27(2):255-61.

54. Serruys PW, van Hout B, Bonnier H, Legrand V, Garcia E, Macaya C, et al. Randomised comparison of implantation of heparin-coated stents with balloon angioplasty in selec- 
ted patients with coronary artery disease (Benestent II). Lancet. 1998;352(9129):673-81.

55. Theron JG, Payelle GG, Coskun O, Huet HF, Guimarães L. Carotid artery stenosis: treatment with protected balloon angioplasty and stent placement. Radiology. 1996;201(3): 627-36.

56. Roubin G, Yadav S, Iyer S, Vitek J. Carotid stent-supported angioplasty: a neurovascular intervention to prevent stroke. Am J Cardiol. 1996;78(3A):8-12.

57. Diethrich EB, Ndiaye M, Reid DB. Stenting in the carotid artery: initial experience in 110 patients. J Endovasc Surg. 1996;3(1):42-62.

58. Criado FJ, Wellons E, Clark NS. Evolving indications for and early results of carotid artery stenting. Am J Surg. 1997; 174(2):111-4

59. Vozzi CR, Rodriguez AO, Paolantonio D, Smith JA, Wholey $\mathrm{MH}$. Extracranial carotid angioplasty and stenting. Initial results and short-term follow-up. Tex Heart Inst J. 1997;24 (3):167-72.

60. Yadav JS, Roubin GS, lyer $S$, et al. Elective stenting of the extracranial carotid arteries. Circulation. 1997;95(2):376-81.

61. Wholey $\mathrm{MH}$, Jarmolowski CR, Eles G, Levy D, Buecthel J. Endovascular stents for carotid artery occlusive disease. J Endovasc Surg. 1997;4(4):326-38.

62. Henry M, Amor M, Klonaris C, Henry I, Masson I, Chati Z, et al. Angioplasty and stenting of the extracranial carotid arteries. Tex Heart Inst J. 2000;27(2):150-8.

63. Teitelbaum GP, Lefkowitz MA, Giannotta SL. Carotid angioplasty and stenting in high-risk patients. Surg Neurol. 1998;50 (4):300-12.

64. Waigand J, Gross CM, Uhlich F, Kramer J, Tamaschke C, Vogel $\mathrm{P}$, et al. Elective stenting of carotid artery stenosis in patients with severe coronary artery disease. Eur Heart J. 1998;19(9):1365-70

65. Bergeron P, Becquemin JP, Jausseran JM, Biasi G, Cardon JM, Castellani L, et al. Percutaneous stenting of the internal carotid artery: the European CAST I Study. Carotid Artery Stent Trial. J Endovasc Surg. 1999;6(2):155-9.

66. Roubin GS, New G, lyer SS, Vitek JJ, Al-Mubarak N, Liu $\mathrm{MW}$, et al. Immediate and late clinical outcomes of carotid artery stenting in patients with symptomatic and asymptomatic carotid artery stenosis: a 5-year prospective analysis. Circulation. 2001;103(4):532-7.

67. Phatouros CC, Higashida RT, Malek AM, Meyers PM, Lempert $\mathrm{TE}$, Dowd CF, et al. Clinical use of stents for carotid artery disease. Neurol Med Chir (Tokyo). 1999;39(12):809-27.

68. Malek AM, Higashida RT, Phatouros CC, Lempert TE, Meyeus PM, Smith WS, et al. Stent angioplasty for cervical carotid artery stenosis in high-risk symptomatic NASCET-inelegible patients. Stroke. 2000;31(12):3029-33.

69. Malek AM, Higashida RT, Phatouros CC, Lempert TE, Meyeus PM, Gress DR, et al. Treatment of posterior circulation ischemia with extracranial percutaneous balloon angioplasty and stent placement. Stroke. 1999;30(10):2073-85.

70. Malek AM, Higashida RT, Phatouros CC, Lempert TE, Meyeus PM, Smith WS, et al. Endovascular management of extracranial carotid artery dissection achieved using stent angioplasty. AJNR Am J Neuroradiol. 2000;21(7):1280-92.

71. Wholey M, Eles G. Clinical experience in cervical carotid artery stent placement. Carotid Neurovasc Int. 1998;50(4):301-6.

72. North American Symptomatic Carotid Endarterectomy Trial Collaborators. Beneficial effect of carotid endarterectomy in symptomatic patients with high-grade carotid stenosis. N Engl J Med. 1991;325(7):445-53.

73. Jordan WD Jr, Schroeder PT, Fisher WS, Mc Dowell HA A comparison of angioplasty with stenting versus endarterectomy for the treatment of carotid artery stenosis. Ann Vasc Surg. 1997; 11(1):2-8.

74. Higasida RT, Malek AM, Phatouros CC. Stenting and angioplasty for cerebrovascular disease. In: Fischer M, Fieschi C, eds. Prevention of ischemic stroke. London:Martin Dunitz Publishers;1999. p.231-52.

75. Phatouros CC, Higashida RT, Lefler JE. Angioplasty and stent placement in the extracranial circulation. In: Marks MP, Do HM, eds. Endovascular and percutaneous therapy of the brain and spine. Philadelphia:Lippincott Williams \& Wilkins;2002. p.43-7.

76. Phatouros CC, Higashida RT, Malek AM, Meyeus PM, Lempert $\mathrm{TE}$, Dowd CF, et al. Carotid artery stent placement for atherosclerotic disease: rationale, technique, and current status. Radiology. 2000;217(1):26-41.

77. Endovascular versus surgical treatment in patients with carotid stenosis in the Carotid and Vertebral Artery Transluminal Angioplasty Study (CAVATAS): a randomised trial. Lancet. 2001;357(9270):1729-37.

78. Alberts MJ, McCabb R, Smith TP. A randomized trial of carotid stenting vs endarterectomy in patients with symptomatic carotid stenosis. J Neurovasc Dis. 1997;2:228-34.

79. Hobson RW $2^{\text {nd }}$. CREST (Carotid Revascularization Endarterectomy versus Stent Trial): background, design, and current status. Semin Vasc Surg. 2000;13(2):139-43.

80. Hobson RW $2^{\text {nd }}$, Brott $T$, Ferguson R, Roubin G, Moore W, Kuntz $R$, et al. CREST: carotid revascularization endarterectomy versus stent trial. Cardiovasc Surg. 1997;5(5):457-8.

81. Theron J, Guimarães L, Coskun $\mathrm{O}$, Sola T, Martin JB, Rufenacht DA. Complications of carotid angioplasty and stenting. Neurosurg Focus. 1998;5(6):e4.

82. Henry M, Amor M, Masson I, Henry I, Tzvetanov K, Chat $Z$, et al. Angioplasty and stenting of the extracranial carotid arteries. J Endovasc Surg. 1998;5(4):293-304.

83. Yadav JS, Wholey MH, Kuntz RE, Fayad P, Katzen BT, Mishkel GJ, et al. Protected carotid-artery stenting versus endarterectomy in high-risk patients (SAPPHIRE). N Engl J Med. 2004;351(15):1493-501.

84. Mas JL, Chatellier G, Beyssen B, Branchereau A, Moulin T, Becquemin JP, et al. Endarterectomy versus stenting in patients with symptomatic severe carotid stenosis. $N$ Engl J Med. 2006;355(16):1660-71.

85. Ringleb PA, Allenberg J, Bruckmann $\mathrm{H}$, Eckstein $\mathrm{HH}$, Fraedrich G, Hartmann M, et al. 30 day results from the SPACE trial of stent-protected angioplasty versus carotid endarterectomy in symptomatic patients: a randomised non-inferiority trial. Lancet. 2006;368(9543):1215-6. 